\title{
EFECTO DE OCHO CLASES DE EXPRESIÓN CORPORAL EN EL ESTADO DE ÁNIMO Y AUTOCONCEPTO GENERAL DE JÓVENES UNIVERSITARIOS
}

\author{
Vivian Rodríguez Barquero \\ Docente de la Escuela de Educación Física y Deportes \\ Universidad de Costa Rica \\ San José, Costa Rica \\ Gerardo Alonso Araya Vargas \\ Docente de la Escuela de Educación Física y Deportes \\ Universidad de Costa Rica \\ San José, Costa Rica
}

Recibido 25-V-2009 • Aceptado 18-IX-2009 • Corregido 18-XI-2009

\begin{abstract}
Resumen: El propósito del estudio fue indagar sobre los efectos psicológicos de la expresión corporal en estudiantes de la Universidad de Costa Rica. Se trabajó con 35 jóvenes de dos grupos: 17 de taller de teatro y 18 de apreciación de teatro. Su promedio de edad: hombres $18,78 \pm 1,42$ y mujeres $18,52 \pm 1,91$ años. Los sujetos de taller realizaron ocho sesiones de expresión corporal de dos horas, una vez por semana por ocho semanas. Los sujetos de apreciación recibieron ocho clases magistrales sobre teatro, de dos horas, una vez por semana por ocho semanas. Las mediciones del estado de ánimo se realizaron por medio del perfil de estados de ánimo (P.O.M.S.) pre y post de la primera, la cuarta y octava sesión. Las mediciones del autoconcepto general se realizaron previo al inicio de la primera sesión (Pre), al final de la cuarta sesión (Test), y al final de la octava sesión (Post-test). Como resultados se encontraron mejoras significativas en los estados de ánimo de tensión y vigor, así como en la variable de inhibición-empatía-desconfianza. Se concluye que la expresión corporal puede influir positivamente sobre estados de ánimo y el autoconcepto de estudiantes de nivel universitario, durante su primer ciclo en la Universidad.
\end{abstract}

Palabras clave: Expresión corporal, teatro, estados de ánimo, autoconcepto general.

\section{Introducción}

\section{Expresión corporal}

La expresión corporal es elemento indispensable en la comunicación y el aspecto social de la persona; en palabras de las autoras Stokoe y Harf (1992), es una conducta que existe desde siempre en todo ser humano, es un lenguaje paralingüístico por medio del cual este se expresa por medio de sí mismo, reuniendo, en el propio cuerpo, el mensaje y el canal, el contenido y la forma. Por esta razón, ha sido parte de la educación por medio del movimiento. Bolaños (1986) explica que su estudio consiste en el desarrollo de reacciones eficaces, eficientes y expresivas en el aspecto motor de un individuo integral que piensa, siente y comparte.

La implementación de este concepto como técnica se da a partir de 1944, 


\begin{abstract}
The purpose of this study was to investigate the psychological effects of body expression in students from the University of Costa Rica. Thirty-five young people from two groups: 17 from Theater Workshop and 18 from Theater Appreciation. Average age: men $18.78 \pm 1.42$ and $18.52 \pm 1.91$ age women. The first subjects performed eight corporal expression sessions of two hours once a week for eight weeks. The second subjects received eight lectures on theater appreciation, two hours once a week for eight weeks. The measurements of mood were made using the profile of mood states (POMS) pre and post the first, fourth and eighth session. Measurements of self-concept were conducted prior to the commencement of the first session (Pre), at the end of the fourth session (Test), and at the end of the eighth session (Post-test). There were significant improvements in mood states of tension and vigor and in the variable of inhibition-empathy-distrust. It is concluded that the body expression can have a positive impact on moods and self-concept of college students during their first semester on University.
\end{abstract}

Key words: Body expression, drama, moods, selfconcept. según Penchansky (1998), cuando se le denominó "Educación por el arte". La diferencia principal con otras técnicas de danza fue su aspiración democratizante; es decir, constituye una danza al alcance de todos y todas. Por poseer características artísticas y sociológicas, permite expresar de manera creativa estados emocionales, utilizando el cuerpo, los movimientos y ejercitando los sentidos. Relacionado con esto, Brehm y Kampfe (1997) plantean que la expresión corporal, como disciplina del movimiento, envuelve tanto movimiento del cuerpo como el despertar y la conciencia de la creatividad, con lo cual se satisfacen dos necesidades humanas básicas: la de moverse y la de crear.

En síntesis, en la expresión corporal la persona practicante pone atención a lo que siente su cuerpo y se deja llevar por esa sensación del movimiento, que enfatiza en cómo se mueve el cuerpo y cómo lo hace en el espacio, por medio de una cualidad emocional y de sensaciones, guiado por la creación del movimiento. Además aprende lo que Sheets-Johnstone (1981) llama "Pensar en Movimiento", proceso en que las sensaciones se vuelven movimiento y el movimiento se vuelve sensación.

Esta disciplina trabaja directamente con la posibilidad de imaginar, lo que provoca en la persona practicante una sensación de autoeficacia corporal y a la vez de conexión con los otros; es decir, se produce lo que Erickson (2004) denomina una dialéctica que busca la libertad de la persona y su unión con el grupo. Dentro de esta característica socializadora de la expresión corporal, los autores Brehm y Kampfe (1997) y Mays (2000) agregan que mediante la expresión del movimiento se ayuda a la persona a conectarse consigo misma, y por medio de esta conexión se comunica con los otros. De manera que al darle importancia a la individualidad de la expresión se promueve, según ellos, la socialización. 


\section{Expresión corporal: Teatro-Danza}

Para entender los aspectos que trabaja esta técnica, es necesario mencionar que la expresión corporal es parte del arte escénico o dramático; por lo tanto, posee dos instrumentos primordiales: la voz y el cuerpo. Según Ratliff (1997), Van`t Hof (2002) y Williams (2001), los ejercicios que se practican en esta disciplina se desarrollan por la sugestión, la motivación, la autoexpresión, la exploración creativa, la observación (propia y del otro), la imaginación corporal, la cooperación; los que también son ejercicios importantes en la instrucción del arte escénico.

Dentro de los beneficios de la expresión corporal, identificados por diferentes autores están: ofrecer a la persona una inteligencia kinética (uso de todo el cuerpo o sus partes), así como un incremento de sus habilidades de observación, físicas, cognitivas, sociales y afectivas, desarrollo del pensamiento abstracto, su autoestima, su pensamiento crítico, creatividad motora, concentración en las tareas del movimiento y análisis de problemas. Se expande, además, su conciencia interpersonal y se aprende el valor de la diferencia individual (Chen y Cone, 2003; Erickson, 2004; Jensen, Rechis y Luna, 2001; Smutny, 2002; Wang, 2003).

\section{Improvisación teatral}

Por último, cabe mencionar a la improvisación como elemento primordial del trabajo a realizar en la expresión corporal. Sus principales objetivos son la comunicación y sintonía grupal, en el momento; así como el juego y la desinhibición. Gravel (1987) define la improvisación teatral como un juego que se sirve de los principios propios del teatro, agregándole sus propias reglas.

Gravel es el creador del match de improvisación y de la L.N.I. (Liga Nacional de Improvisación) de Canadá, la primera del mundo. La impro es una técnica que intenta unir el teatro y el deporte, en un juego donde el improvisador o la improvisadora se entrena para crear historias en el momento, jugando roles de dirección, actuación y escritura al mismo tiempo. La regla principal de este juego es la no censura de la imaginación. Por esto, dentro de las clases de expresión corporal se realiza la improvisación, aspecto esencial para trabajar la comunicación, la aceptación y conciencia de la imaginación.

Ahora bien, en el campo de instrucción y práctica de la expresión corporal, los estudios experimentales son prácticamente nulos; el único estudio encontrado es de Gelder, Snyder, Greve, Gerard y Hadjikhani (2004), quienes realizaron una investigación sobre la influencia de los movimientos corporales expresivos (expresión de emociones con el cuerpo), en la neurobiología de la conducta emocional y encontraron una relación entre la expresión del cuerpo y la activación del cerebro.

Por otra parte, dentro del campo de la improvisación teatral, tampoco se hallaron estudios, salvo por una investigación previa de Rodríguez y Araya (2007), en la cual se analizó el efecto de la improvisación en el estado de ánimo de jóvenes costarricenses privados de libertad. Entre los resultados se observaron disminuciones significativas en las variables de tensión, depresión, fatiga y cólera, tras un tratamiento con improvisación teatral. Por lo tanto, son necesarios estudios como este, para entender más los efectos psicológicos de esta actividad.

Después de la búsqueda bibliográfica, se llegó a la conclusión de que lo investigado a nivel artístico de esta técnica es poco y lo que se ha realizado no está documentado o publicado (experiencias personales), por lo que se vuelve primordial su estudio para ahondar en los beneficios que posee. En este sentido, por ejemplo, Donnelly (2005) afirma que es importante tomar en cuenta que "el arte se está practicando actualmente, como 
un medio para la autoexpresión, para tocar el lado creativo de la persona, como una alternativa de curación; y no tanto para el desarrollo de un talento artístico especial" (p. 3). Es necesario, entonces, verificar esta afirmación en la práctica con estudios experimentales que brinden información clara de los efectos del arte como terapia.

\section{Autoconcepto}

De acuerdo con Sonstroem (1998), el autoconcepto es una configuración organizada de la percepción del propio ser y aceptada por la conciencia. Puede ser visto como un retrato personal o una autodescripción, por lo tanto, de este depende una adecuada interacción social.

En la perspectiva de Harris (1976), se puede relacionar el desarrollo del autoconcepto con la forma en la que se es percibido por los demás mediante la interacción social, y también se le relaciona con la percepción del propio cuerpo. Así, es de esperar que el deporte y otras formas de actividad física aporten notables oportunidades para el desarrollo y consolidación del autoconcepto.

Uno de los pocos estudios realizados en Costa Rica sobre autopercepción y actividad física fue llevado a cabo por Fallas (2001), quien adaptó al español una escala propuesta por Erdman. Para ello, se aplicó la escala a jóvenes costarricenses estudiantes de secundaria y se determinó su estructura factorial; luego, se analizó cuáles eran las dimensiones más importantes relativas al nivel de actividad física de los sujetos (varones y mujeres). De hecho, de este estudio proviene el instrumento principal utilizado en la presente investigación, para medir el autoconcepto.

Valerín y Sánchez (2004) realizaron un estudio experimental, donde se continuó esta línea de investigación con adolescentes costarricenses. Estas investigadoras tenían el propósito de determinar el efecto de un programa de actividad física-recreativa sobre el autoconcepto físico y general, la práctica de actividad física en el tiempo libre y la autoeficacia para la práctica de actividad física. El grupo de adolescentes asistía a un colegio urbano-marginal, el cual fue la sede del programa, y este se realizó durante el tiempo lectivo regular. En el estudio participaron 78 adolescentes (varones y mujeres), con edades entre los 12 y 17 años. Entre los resultados, se encontró que el programa de actividad física-recreativa tuvo efectos positivos en el autoconcepto general (dimensión yo social), en el autoconcepto físico (percepción de la resistencia), en la práctica de actividad física en el tiempo libre y en la autoeficacia para la práctica de actividad física.

En sujetos universitarios son escasos los estudios donde se relacione la práctica de algún tipo de actividad física sistematizada con las autopercepciones. En Costa Rica, el único antecedente lo constituye el estudio realizado por Araya et al. (2004), quienes evaluaron varias dimensiones del autoconcepto en 585 estudiantes matriculados en cursos de Actividad Deportiva de la Universidad de Costa Rica, durante el año 2003. Encontraron que la participación en los cursos de actividad deportiva de la universidad afectaba positivamente algunos componentes del autoconcepto físico del estudiantado.

Por lo tanto, hay evidencias promisorias con respecto a posibles beneficios para el estudiantado en variables psicosociales por participar en programas de actividad física sistematizada. No obstante, las actividades que tengan como propósito la promoción de la expresión de sentimientos y demás aspectos de la subjetividad, mediante el movimiento, no son parte de la oferta regular de cursos de actividad deportiva para la población universitaria y con base en la revisión de estudios previos, los efectos de esta modalidad de actividad (la expresión corporal) podrían ser distintos a los ya observados en otros tipos de actividades.

En resumen, el propósito de este estudio es dilucidar los efectos en el estado de ánimo y el autoconcepto de jóvenes universitarios, de un curso en el que 
se promueve la expresión corporal. Los análisis se centrarán en los efectos agudos (por sesión) y crónicos (a lo largo del curso). También, se tomará en cuenta al sexo como variable y se realizará la comparación entre el curso de expresión corporal y una clase de iguales características, pero en la que no se realiza ningún trabajo físico sistematizado ni vigoroso.

\section{Metodología}

\section{Sujetos}

Participaron 35 jóvenes universitarios de primer ingreso de la Universidad de Costa Rica, de los cuales 17 eran estudiantes del curso "Taller de Teatro" (expresión corporal, 11 mujeres y 6 hombres) y 18 eran estudiantes de "Apreciación de Teatro" (clases teóricas magistrales, 10 mujeres y 8 hombres). Ninguno de los grupos tenía integrantes que hubiesen hecho antes teatro o clases de expresión corporal.

Por sexo, la edad promedio fue en los hombres $18,78 \pm 1,42$ años y en las mujeres 18,52 $\pm 1,91$ años. Por grupo, la edad promedio de las personas integrantes del taller de teatro fue $18,41 \pm 1,28$ años y en el grupo de apreciación, 18,83 \pm 2,06 años. No se encontró diferencia significativa en la edad de los sujetos, según grupo o sexo $\left(\mathrm{F}_{\text {grup o }}=0,131 ; \mathrm{F}_{\text {sexo }}=0,225 ; \mathrm{F}_{\text {grupo } \mathrm{X} \text { sexo }}=\right.$ $2,186$; todas con $\mathrm{p}>0,05)$. Por lo tanto, la edad no fue una variable que pudiera haber afectado los resultados.

\section{Instrumentos}

Escala del perfil de estados de ánimo (POMS, Profile of Mood States). McNair, Lorr y Droppleman (1971, 1981, 1992) desarrollaron este instrumento para valorar el estado anímico de pacientes ambulatorios de centros de salud mental, pero progresivamente se vio la utilidad del perfil que se podía obtener con esta escala para aplicarlo en deportistas. Morgan (1979, 1985, 1991) fue el primero en aplicar este instrumento al campo de la Psicología del Deporte y con base en el POMS desarrolló el modelo de salud mental (Morgan, Brown, Ragling, O'Connor y Ellickson, 1987; Morgan, Costill, Flynn, Ragling y O'Connor 1988), que ha mostrado su validez para discriminar entre atletas y no atletas. También, se ha demostrado la utilidad del POMS para predecir el rendimiento en ciertas disciplinas deportivas (Beedie, Terry y Lane, 2000). Se usó la adaptación española corta (Fuentes, García-Merita, Meliá y Balaguer, 1994) de 15 ítems cuantificados mediante una escala tipo Likert. Esta versión mide cinco estados anímicos (tensión, depresión, fatiga, cólera y vigor) y goza de validez y confiabilidad en español. Además, esta versión corta es recomendada cuando se valora el efecto agudo de una sesión, para controlar el posible efecto de aprendizaje.

Escala de autoconcepto general de Erdmann (Fallas, 2001). Este instrumento consta de 36 ítems, los cuales se cuantifican mediante una escala tipo Likert. Según análisis factoriales aplicados en población costarricense en estudios previos (Fallas, 2001), esta escala valora seis dimensiones, las cuales se denominaron como: yo general, yo personal, optimismo/ pesimismo, optimismo/actividad física, yo social, inhibición/empatía/desconfianza. Este instrumento se ha utilizado con éxito en un estudio experimental previo (Valerín y Sánchez, 2004), realizado en adolescentes que recibieron un programa de actividades vigorosas y lúdicas en su colegio.

\section{Procedimientos}

Como se anticipó, al describir las características generales de los sujetos, se crearon dos grupos. A cada uno se le aplicó un curso diferente, Taller (práctico) y Apreciación (teórico), los cuales se describirán más adelante. Aún así, se tuvo el cuidado de 
que ambos grupos fuesen atendidos por el mismo docente. Los grupos se crearon con base en la matrícula que se tuviera, por lo que no fue posible asignar aleatoriamente a los participantes a cada grupo.

Las actividades realizadas en cada grupo, fueron las siguientes:

Grupo de taller de teatro: este grupo realizó ocho sesiones de expresión corporal de dos horas cada una, durante ocho semanas (se hacía una clase por semana), con el siguiente orden de actividades:

- Calentamiento físico: ejercicios de estiramiento, expresión corporal, reacción, coordinación mental y física. Además, ejercicios con utilización de la voz y de la expresión facial.

- Ejercicios de imaginación corporal: juegos teatrales de invención de personajes, situaciones, historias y juegos de introducción a la comunicación y confianza grupal.

- Ejercicios de improvisación teatral, con énfasis en la expresión corporal: improvisaciones hechas en grupo sin otorgar ningún tiempo de preparación. Las improvisaciones partían de temas, palabras, situaciones y estímulos propuestos por la docente.

Grupo de apreciación: este grupo realizó ocho clases magistrales de dos horas (se hacía una clase por semana) durante ocho semanas (de modo que ambos grupos trabajaron un tiempo similar), con el siguiente orden de actividades:

- Exposiciones de la docente: introducción de temas técnicos del teatro, cómo funciona la actuación, en qué consiste la dirección, cómo es la relación actores-público, cuál es el papel del espectador en el teatro, tipos de espectáculos y técnicas teatrales, espectáculos nacionales.
- Discusiones sobre algún tema: después de desarrollado el tema por la docente, se realizan discusiones sobre preguntas hechas al estudiantado.

\section{Análisis estadístico}

Se realizó estadística descriptiva: con promedios y desviaciones estándar. Como estadística inferencial, se aplicó un análisis de varianza (ANOVA) de dos vías (grupos x sexo), para comparar la edad y se aplicó un ANOVA de cuatro vías (grupo x sesión $\mathrm{x}$ medición $\mathrm{x}$ sexo) para comparar cada estado anímico (tensión, depresión, fatiga, vigor, cólera) en los niveles de las variables independientes correspondientes. Además, se aplicó un ANOVA de tres vías (medición $\mathrm{x}$ grupo $\mathrm{x}$ sexo) para comparar las dimensiones de autoconcepto (yo general, yo personal, optimismo-pesimismo, optimismo-actividad física, yo social e inhibición-empatía-desconfianza) y se realizó el análisis de efectos simples (Keppel, 1982), cuando se ameritaba. Todo se analizó por medio del programa SPSS (versión 8.0 para Windows).

\section{Resultados}

\section{Estados anímicos}

Se encontró interacción significativa en la variable tensión, entre sesión y grupo $(\mathrm{F}=5,772 ; \mathrm{p}<0,01)$. El análisis de efectos simples de la interacción (sesión $\mathrm{x}$ grupo) mostró que no existió diferencia significativa en la tensión entre las sesiones para el grupo de taller (expresión corporal); es decir, que la tensión en este grupo se mantuvo estable a lo largo de su tratamiento. Sin embargo, el grupo de apreciación (clases teóricas magistrales) presentó un promedio significativamente más alto en la sesión ocho $(\mathrm{F}=15,610 ; \mathrm{p}<0,01)$, que el presentado en las sesiones uno y cuatro, es decir, aumentó su nivel de tensión al final del estudio. Esto se ilustra en el gráfico 1. 


\section{Gráfico 1}

Interacción entre sesiones y grupo para la variable tensión

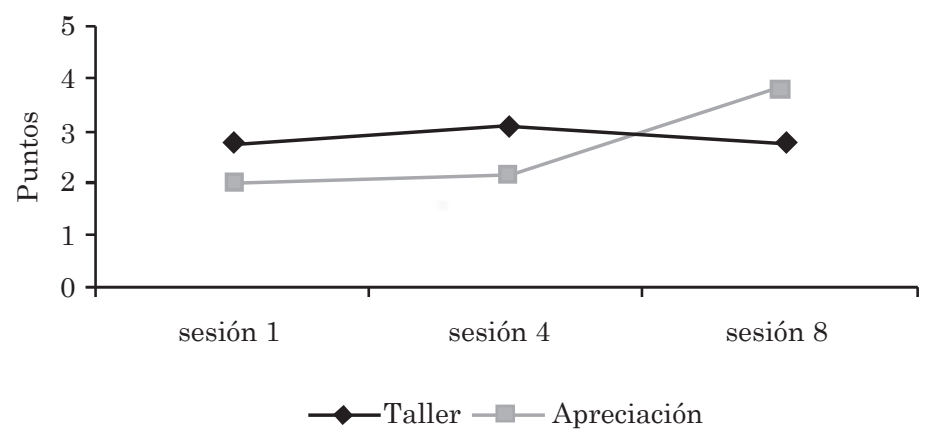

Fuente: Elaboración propia.

Se encontró interacción significativa entre sesión y medición ( $\mathrm{F}=3,881 ; \mathrm{p}<0,05)$ en la variable de depresión. El análisis de efectos simples de la interacción (sesión $\mathrm{x}$ medición) mostró que no hubo diferencias en los niveles de depresión entre las tres sesiones evaluadas. Sin embargo, en la sesión uno y la sesión ocho (final), se encontró una disminución significativa de la depresión. Al no haberse encontrado interacción con la variable "grupo", esto indica que estas disminuciones se dieron por igual en ambos grupos. Esto se ilustra en el gráfico 2.

Gráfico 2

Interacción entre sesiones y mediciones para la variable depresión

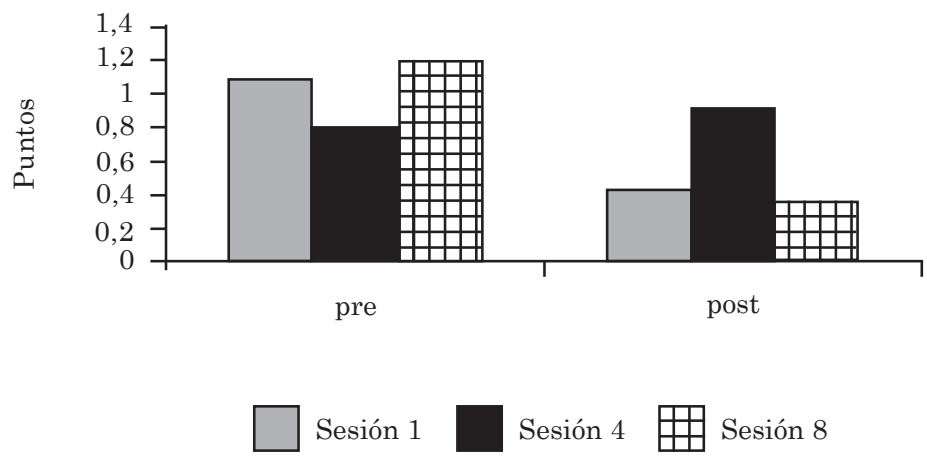

Fuente: Elaboración propia.

Además, se encontró diferencia significativa entre grupos $(\mathrm{F}=4,627 ; \mathrm{p}$ $<0,05)$ con respecto a la variable fatiga. En general, el grupo de Taller (expresión corporal) mantuvo siempre niveles de fatiga más bajos que los del grupo de apreciación.
No se encontró ninguna otra diferencia significativa en esta variable.

Por otra parte, se encontró interacción significativa entre medición y grupo $(\mathrm{F}=$ $25,444 ; \mathrm{p}<0,01)$ en la variable de vigor. Cabe recordar aquí que la variable medición 
constaba de dos niveles de comparación: pre (inicio de cada sesión) y post (final de cada sesión). En la interacción entre grupo y medición, se compara el promedio general que se obtiene de los pre-test de tres sesiones (primera, cuarta y octava), con el promedio general de los post-test de esas sesiones y esa comparación pre frente a post, se hace entre los dos grupos. Todas esas comparaciones se desglosan con el análisis de efectos simples de la interacción (medición x grupo). Este análisis mostró que existió un aumento significativo del vigor entre el pre-test y post-test en el grupo de Taller (expresión corporal). En el caso del grupo de Apreciación, no se presentó este efecto. También se observó que no existió diferencia significativa en el promedio de vigor de ambos grupos en el pre-test; no obstante, sí se encontró que el grupo de Taller (expresión corporal) presentó más vigor que el grupo de Apreciación en el post-test. Esto significa que en las sesiones primera, cuarta y octava (final) del estudio, ambos grupos iniciaban con niveles de vigor similares, pero al final de esas sesiones, siempre ocurría que el grupo de Taller de Teatro (expresión corporal) mejoraba su vigor, mientras que el grupo de Apreciación (clases magistrales y teóricas) se mantenía igual que al inicio. Esto se observa en el gráfico 3 .

Gráfico 3

Interacción entre mediciones y grupos para la variable vigor

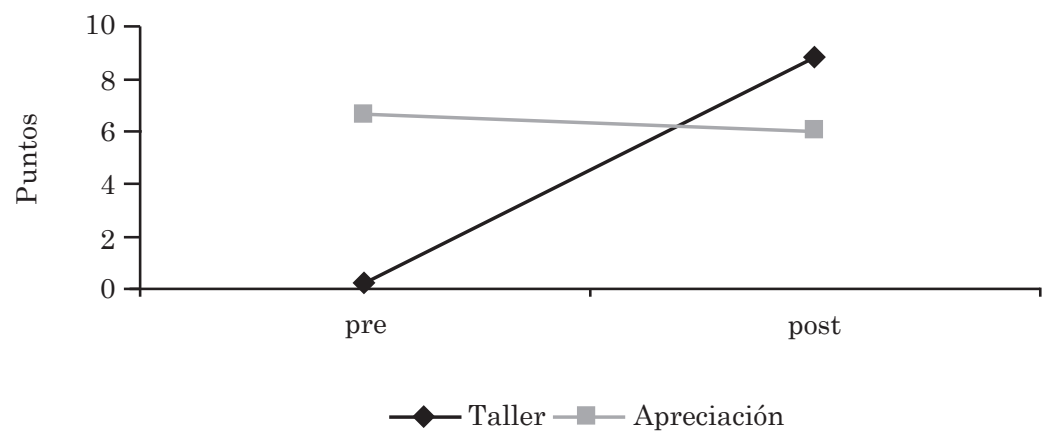

Fuente: Elaboración propia.

Finalmente, se encontró diferencia significativa entre mediciones $(\mathrm{F}=5,695$; $\mathrm{p}<0,05$ ) en la variable de cólera. Los niveles de cólera independientemente del grupo, sesión y sexo, siempre fueron más bajos en el post-test, pero al no existir interacción significativa con la variable "grupo", eso indica que el tratamiento no influyó significativamente sobre esta variable.

\section{Autoconcepto}

Se encontró diferencia significativa entre sexos $(\mathrm{F}=5,651 ; \mathrm{p}<0,05)$ en la variable yo general. Los niveles del autoconcepto general del yo fueron mayores en las mujeres que en los hombres. Según Fallas (2001), un puntaje alto en esta variable se interpreta como negativo, es decir, las mujeres en este caso presentaron menor satisfacción con $\mathrm{su}$ yo general (autoestima, autoeficacia, autoconfianza) que los hombres. Esto se presentó independientemente del grupo, sesión o medición; por lo tanto, el tratamiento no afectó esta variable. Por otra parte, no se encontró diferencias o interacciones significativas en los puntajes de yo personal, así como en los puntajes de optimismo-pesimismo y yo social.

Por otro lado, se encontró interacción significativa entre medición 
$\mathrm{y} \operatorname{sexo}(\mathrm{F}=3,950 ; \mathrm{p}<0,05)$ en los puntajes de optimismo-actividad física (mayor empatía y autoconfianza hacia los deportes). El análisis de efectos simples de la interacción (medición x sexo) mostró que no se encontró diferencia significativa en el promedio general de las tres sesiones evaluadas. A pesar de lo anterior, se encontró diferencia significativa en la octava sesión, entre ambos sexos, donde los hombres presentaron un puntaje significativamente más alto en comparación con las mujeres. Esto se observa en el gráfico 4 .

\section{Gráfico 4}

Interacción entre mediciones y sexo para la variable de Optimismo-actividad física

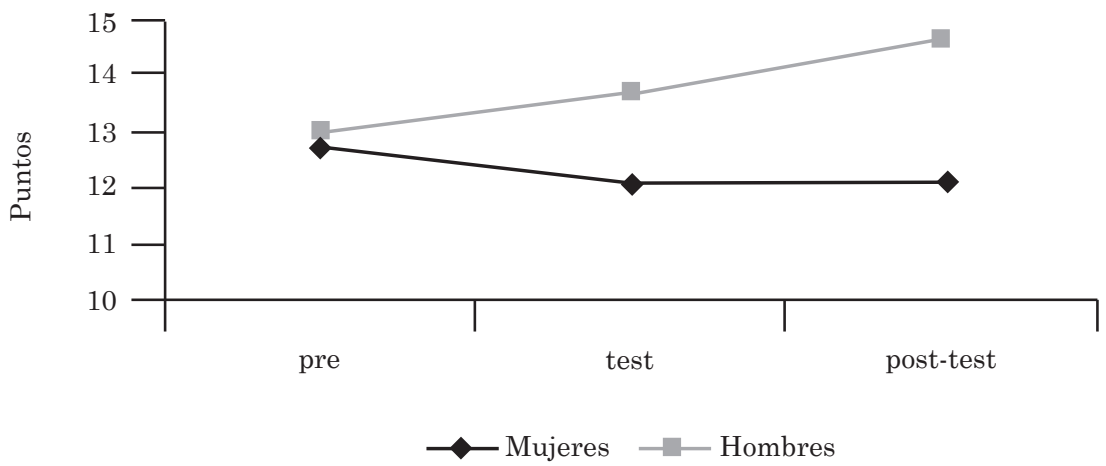

Fuente: Elaboración propia.

Por último, se encontró interacción significativa entre medición y grupo $(\mathrm{F}=3,579 ; \mathrm{p}<0,05)$ en la variable de inhibición, empatía y desconfianza (empatía social). El análisis de efectos simples de la interacción (medición x grupo) mostró que hubo diferencia significativa entre grupos, en la cuarta sesión, donde el grupo de taller (expresión corporal) tuvo un puntaje significativamente más alto que el grupo de apreciación. Esto se observa en el gráfico 5.

\section{Gráfico 5}

Interacción entre mediciones y grupo para la variable de inhibición, empatía y desconfianza

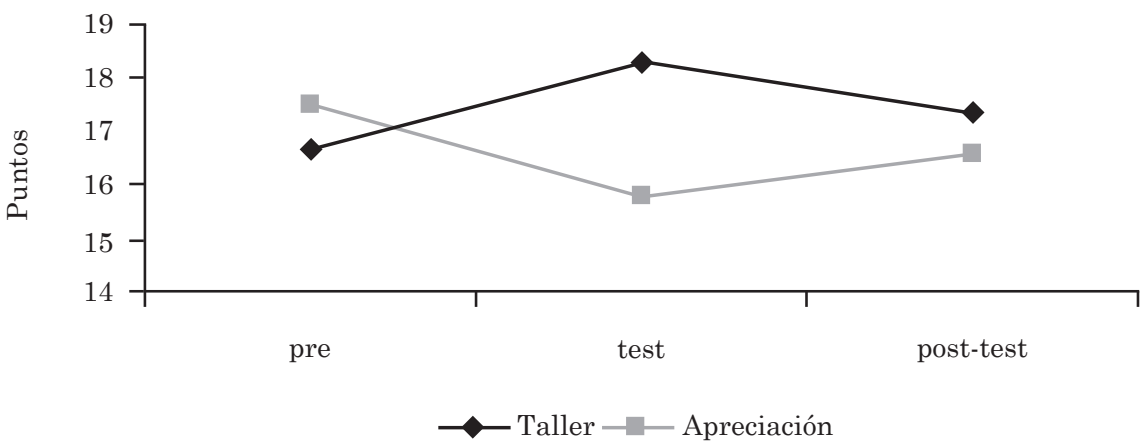

Fuente: Elaboración propia. 


\section{Discusión}

Los resultados encontrados en este estudio abren un campo de investigación que sería interesante profundizar. Al ser un estudio pionero en esta área, se pueden observar tendencias importantes de interpretar. Por ejemplo, se muestra un aumento de la tensión en el grupo de Apreciación (clase teórica magistral), a diferencia del grupo de Taller (clase de expresión corporal), ya que para la cuarta y octava sesión en el grupo de apreciación, se aumentó la tensión. Esta interacción puede atribuirse a la demanda de trabajos y exámenes de tipo intelectual que se requiere del estudiantado universitario a partir de la mitad del semestre (sesiones cuatro a ocho del estudio), lo que se reflejó claramente en el grupo de Apreciación, a diferencia del grupo de Taller, que al estar en contacto con trabajos de tipo físico práctico (juego y uso de la imaginación), no presentó este aumento.

Parece ser que el trabajo práctico convierte a la clase de expresión corporal en una especie de descanso para el estudiantado, ya que a pesar de tener las mismas demandas de cualquier curso universitario, las personas participantes en el de Teatro (expresión corporal) mostraron la posibilidad de relajarse. A diferencia de lo sucedido a los participantes del curso teórico (Apreciación), cuyo nivel de tensión se mantuvo bajo y estable a lo largo de las ocho semanas del estudio. Este resultado concuerda con lo planteado por Erickson (2004) sobre los efectos de este tipo de trabajo físico: una sensación de confianza y relajación.

Además se determinaron dos interacciones que pueden deberse a factores externos ajenos al tratamiento. Por ejemplo, la interacción entre sesión y medición en la variable depresión puede deberse al estilo de enseñanza del docente de ambos grupos. Por otro lado, la interacción entre medición y sexo, encontrada en la variable optimismo y actividad física, muestra un aumento de optimismo hacia la actividad física en el caso de los hombres, lo cual se puede deber a factores fuera de control, como actividades paralelas que estuviesen realizando los sujetos de tipo recreativo y deportivo, ya que en las actividades dentro del estudio hubo una adecuada participación y actitud de parte de ambos sexos.

Pero para ambas variables (depresión y optimismo y actividad física), no hubo interacción entre las variables "mediciones" y "grupo". Por lo tanto, lo que acontecía en cada grupo no logró afectar significativamente esas variables. Sin embargo, es probable que esas variables, para la población estudiantil participante en el estudio, no presentaban niveles iniciales que tuvieran una gravedad tal que requirieran de mejora. En el caso de la depresión, sus niveles fueron siempre bajos mientras que los niveles de optimismo y actividad física siempre se mantuvieron dentro del puntaje medio de la escala respectiva y solo se observó diferencias ligadas al sexo en esta variable del autoconcepto.

Un efecto importante del tratamiento se pudo observar en el aumento significativo del vigor de pre-test a post-test, en el caso del grupo de taller de teatro (expresión corporal). Esto podría explicarse por el aumento progresivo de la energía demandada por la expresión corporal para poder responder a las exigencias del medio (hablar en público, exponerse a personas desconocidas, expresar sentimientos y pensamientos personales frente a extraños, etc.), lo cual maximiza así los efectos energéticos que de por sí posee el teatro como trabajo físico. Este parece ser un efecto que se aumenta con el tiempo, ya que se va ganando terreno en el campo de la confianza y comunicación grupal y se inicia el disfrute del juego.

Además, se observaron niveles de fatiga significativamente más bajos en el grupo de Taller de Teatro (expresión corporal) en comparación con los promedios del grupo de Apreciación (clases magistrales y teóricas), los cuales se mantuvieron así a lo largo del tratamiento. Esto concuerda con 
resultados previos de Rodríguez y Araya (2007). El trabajo físico que se desarrolla a partir de la expresión corporal demanda un nivel de energía alto de parte del practicante, pero al ser una combinación de trabajo mental, creativo y físico, no lleva al estudiantado a niveles de fatiga. Al contrario, se busca un aumento de energía del practicante. Sin embargo, podría ser que las diferencias observadas se deban al método de creación de los grupos (por matrícula y no aleatoriamente) y no directamente por las diferencias de cada curso. Esto se considera así, al no haberse encontrado interacción significativa entre las variables "mediciones" y "grupo".

Por otra parte, se observó un aumento significativo de la variable de inhibición, empatía y desconfianza, la cual tiene que ver con la empatía para trabajar en equipo $\mathrm{y}$, por lo tanto, para desenvolverse en el trabajo en grupo, además de relacionarse con la autoconfianza y la autoestima que esto requiere. Un puntaje alto en esta variable se interpreta como positivo, es decir, el grupo de taller de teatro (expresión corporal) presentó un efecto positivo en esta variable, en comparación con el grupo de apreciación (clase teórica magistral). En términos de la socialización y de la integración al ámbito universitario (cabe recordar que las personas participantes eran de primer ingreso a la Universidad), estos resultados son muy importantes, pues evidencian cómo mediante la aplicación de actividades de expresión corporal se puede mejorar la autopercepción de la capacidad personal para trabajar en equipo.

Los resultados mencionados antes pueden explicarse porque la expresión corporal y el teatro poseen dinámicas grupales basadas en el desarrollo de la autoconfianza y la autoestima, ya que se enfatizan en la expresión personal sin el uso de patrones modelo y esto proporciona al practicante una sensación de autoaceptación y autoconocimiento de su cuerpo (inteligencia kinestésica). De esto hablan varios autores como Chen y
Cone (2003), Erickson (2004), Jensen et al. (2001), Smutny (2002) y Wang (2003). Dichos autores afirman que esta técnica (expresión corporal) produce un incremento de las habilidades de observación, físicas, cognitivas, sociales y afectivas, de la autoestima; además, expande la conciencia interpersonal y favorece el aprendizaje del valor de la diferencia individual. Esta apreciación es compartida por otros autores como Brehm y Kampfe (1997) y Mays (2000), quienes plantean que este efecto de conexión con uno mismo brinda importancia a la individualidad de la expresión.

Por último, este estudio da indicios acerca de las posibilidades del arte en la mejora del autoconcepto y los estados de ánimo, como lo enfatiza Donnelly (2005), quien plantea la utilización del arte como un medio para la autoexpresión, para tocar el lado creativo de la persona, como una alternativa de curación y no tanto para el desarrollo de un talento artístico especial. Sin embargo, con base en los resultados del presente estudio no se puede verificar dicha afirmación y pretender que la expresión corporal pueda usarse como terapia. No obstante, los resultados aportan evidencias sobre los beneficios potenciales que tienen las técnicas de expresión corporal en el ámbito educativo para mantener estados de ánimo positivos en la población estudiantil a lo largo de un ciclo lectivo y, además, para mejorar su confianza en las demás personas, lo que mejora la asimilación del trabajo en equipo.

Este estudio abre un cuestionamiento sobre los efectos psicológicos de la expresión corporal, pero se vuelve necesario seguir profundizando para tener una idea más clara de las consecuencias los efectos de este tratamiento.

\section{Conclusiones}

La expresión corporal es una técnica física que, por sus contenidos, puede utilizarse, potencialmente, para influir 
en los estados anímicos de estudiantes de educación superior, esto concuerda con investigaciones ya realizadas en otras poblaciones.

Son necesarias más investigaciones en las artes escénicas para profundizar en sus efectos psicosociales en distintas poblaciones.

\section{Referencias bibliográficas}

Araya, G., Meneses, M., Solera, A., Chacón, Y., Moncada, J., y Hernández, J. (2004). Efecto de la participación en cursos de actividad deportiva sobre el autoconcepto en estudiantes de la Universidad de Costa Rica durante los ciclos I y II de 2003. En Escuela de Educación Física y Deportes, Universidad de Costa Rica, Memoria XI Simposio Internacional en Ciencias del Deporte, el Ejercicio y la Salud, 20 al 23 de octubre (p. 33). San José, Costa Rica: el autor.

Beedie, C. J., Terry, P. C., y Lane, A. M. (2000). The profile of mood states and athletic performance: Two metaanalyses [El perfil de estados de ánimo en el rendimiento atlético: Dos meta-análisis]. Journal of Applied Sport Psychology, 12(1), 49-68.

Brehm, M., y Kampfe, C. (1997). Creative dance improvisation: Fostering creative expression, group cooperation, and multiple intelligences [Improvisación de movimiento creativo: Fomenta la expresión creativa, el trabajo en equipo y las inteligencias múltiples]. Ponencia presentada en ChinaU.S. Congreso de Educación. Pekín, República Popular de China, 9-13 julio. (ERIC Servicio de Reproducción de Documentos N ED425401).

Bolaños, G. (1986). Educación por medio del movimiento y expresión corporal.
Costa Rica: Editorial Universidad Estatal a Distancia.

Chen, W., y Cone, T. (2003). Links between children's use of critical thinking and an expert teacher's teaching in creative dance [La relación entre el uso de pensamiento crítico en niños y la enseñanza de movimiento creativo por un experto]. Journal of Teaching in Physical Education, 22(2), 169185.

Donnelly, G. (2005). The arts in health and healing: A personal journey [Las artes en la salud y la curación: Una experiencia personal]. Holist Nurse Practice, 19(1), 1.

Erickson, D. (2004). Creative dance and basketball [Danza creativa y baloncesto]. Teaching Elementary Physical Education, 15(2), 38-40.

Fallas, L. (2001). Autoconcepto general $y$ autoconcepto físico en personas físicamente activas y sedentarias en combinación con el género. Tesis de Licenciatura, Universidad Nacional, Heredia, Costa Rica.

Fuentes, I, García-Merita, M, Melia, J. L., y Balaguer, I. (1994). Formas paralelas de la adaptación valenciana del perfil de ánimo (P.O.M.S). En Universidad de Santiago de Compostela, IV Congreso de Evaluación Psicológica, 21-24 de setiembre. Santiago de Compostela, Galicia, España: el autor.

Gelder, B., Snyder, J., Greve, D., Gerard, G., y Hadjikhani, N. (2004). Fear foster flight: A mechanism for fear contagion when perceiving emotion expressed by a whole body [Promover gestos de huida y miedo: Un mecanismo de contagio del miedo, cuando se percibe la emoción 
expresada con todo el cuerpo]. PNAS, 101(47), 16701-16706.

Gravel, R. (1987). Impro, reflexiones y análisis. Canadá: Edición Leméac.

Harris, D. (1976). ¿Por qué practicamos deporte? Razones somatopsíquicas para la actividad física. Barcelona: Jims.

Jensen, I., Rechis R., y Luna, D. (2001). Early childhood literacy: Programs and strategies to develop cultural, linguistic, scientific and health care literacy for very young children and their families. Year book: Unit 4 Chapter 10 [Alfabetismo en la niñez temprana: Programas y estrategias culturales, lingüisticas, científicas, de promoción de la salud y alfabetización para niños pequeños $y$ sus familias]. Center for Educational Development, Evaluation and Research, Early Childhood, Texas A \& M University, Corpus Christi. (ERIC Servicio de Reproducción de Documentos $\mathrm{N}^{\circ} \mathrm{ED} 468857$ ).

Keppel, G. (1982). Design and analysis a researcher's hand book [Diseño y análisis: Un libro de bolsillo para investigadores] (2a. ed.). United States of America: Prentice Hall.

Mays, D. (2000). Creative dance [Movimiento creativo]. Primary Educator, 6(2), $15-19$.

McNair, D. M., Lorr, M., y Droppleman, L. F. (1971). Manual for the profile of mood states [Manual del perfil de estados de ánimo]. San Diego: Educational and Industrial Testing Services.

McNair, D. M., Lorr, M., y Droppleman, L. F. (1981). Manual for the profile of mood states [Manual del perfil de estados de ánimo]. San Diego: Educational and Industrial Testing Services.

McNair, D. M., Lorr, M., y Droppleman, L. F. (1992). Manual for the profile of mood states [Manual del perfil de estados de ánimo]. San Diego: Educational and Industrial Testing Services.

Morgan, W. P. (1979). Prediction of performance in athletics [Predicción del rendimiento en deportes]. En P. Klavora y J. V. Daniel, (Eds.). Coach, athlete and the sport psychologist [Entrenador, atleta y psicólogo deportivo] (pp. 173-186). Champaign, IL: Human Kinetics Publishers.

Morgan, W. P. (1985). Psychogenic factors and exercise metabolism: A review. [Factores psicogénicos y metabolismo del ejercicio: Una revisión]. Medicine and Science in Sports and Exercise, 17(3), 309-316.

Morgan, W. P. (1991). Monitoring and prevention of the staleness syndrome [Monitoreo y prevención del síndrome de estancamiento]. En Comité Internacional Olímpico, Memorias del Segundo Congreso Mundial de Ciencias del Deporte del COI (pp. 19-23). Barcelona: el autor.

Morgan, W. P., Brown, D. R., Ragling, J. S., O'Connor, P. J., y Ellickson, K. A. (1987). Psychological monitoring of overtraining and stalenees [Monitoreo psicológico del sobreentrenamiento y el estancamiento]. British Journal of Sports Medicine, 21(3), 107-114.

Morgan, W. P., Costill, D. L., Flynn, M.G., Ragling, J. S., y O'Connor, P. J. (1988). Mood disturbance following increased training in swimmers 
[Disturbio anímico posterior al aumento del entrenamiento en nadadores]. Medicine and Science in Sports and Exercise, 20(4), 408-414.

Penchansky, M. (1998). Datos para una historia de la expresión corporal. En Expresión corporal: el cuerpo en movimiento. Colección 0 a 5. La educación en los primeros años. Tomo 7 , (pp. 3-15). Buenos Aires, Argentina: Ediciones Novedades Educativas

Ratliff, G. (1997). Playing the role: Classroom performance approaches to characterization [Actuando el papel: Actuación en la clase para abordar la caracterización]. En $83^{a}$. Reunión Anual de la Asociación Nacional de Comunicación, 19-23 de noviembre. Chicago, EE.UU. (ERIC Servicio de Reproducción de Documentos $\mathrm{N}^{\circ}$ ED415558).

Rodríguez, V., y Araya, G. (2007). Efecto agudo de una sesión de improvisación teatral y de fútbol en el estado de ánimo de adolescentes privados de libertad del Centro de Formación Zurquí. Revista de Ciencias del Ejercicio y la Salud, 5(1), 45-57.

Sheets-Johnstone, M. (1981). Thinking movement [Pensar en movimiento]. Journal of Aesthetics and Art Criticism, 39(4), 399-407.

Smutny, J. (2002). Integrating the arts in to curriculum for gifted students [Integrar las artes en el curriculum de estudiantes super dotados]. (ERIC Servicio de Reproducción de Documentos $\mathrm{N}^{\circ}$ ED470524).

Sonstroem, R. (1998). Physical self concept: Assessment and external validity
[Autoconcepto físico: Medición y validez externa]. Exercise and Sports Sciences Reviews, 26(1), 133-164.

Stokoe, P., y Harf, R. (1992). La expresión corporal en el jardín de infantes (3era. ed.). España: Ediciones Paidós.

Van't Hof, E. (2002). Essence dance: A simple model for improvisation [Danza esencial: Un modelo simple de improvisación]. En Reunión Anual de la Alianza Americana para la Salud, Educación Física, Recreación y Danza, 9-13 de abril. San Diego, CA, EE.UU. (ERIC Servicio de Reproducción de Documentos $\mathrm{N}^{\circ}$ ED464905).

Valerín, J., y Sánchez, D. (2004). Efecto de un programa de actividad física-recreativa en el autoconcepto físico y general, la práctica de actividad física en el tiempo libre y la autoeficacia para la práctica de actividad física de adolescentes de un colegio urbano-marginal. Tesis de Licenciatura en Ciencias del Deporte con énfasis en salud. Escuela Ciencias del Deporte. Facultad de Ciencias de la Salud. Universidad Nacional. Heredia, Costa Rica.

Wang, J. (2003.) The effects of a creative movement program on motor creativity of children ages three to five [Efectos de un programa de movimiento creativo en la creatividad motora de niños de tres a cinco años]. Disertación doctoral. Universidad de Dakota del Sur, EE.UU.

Williams, L. (2001). Creative writing is a moving experience [Escritura creativa una experiencia movilizadora]. Teaching Elementary Physical Education, 12(1), 25-26. 